\title{
Indagine nazionale sulle metodiche per emocoltura in Italia
}

\author{
Antonio Goglio', Pierluigi Nicoletti ${ }^{2}$ \\ 'UO Microbiologia e Virologia, AO Ospedali Riuniti, Bergamo \\ ${ }^{2}$ UO Microbiologia e Virologia, AO Careggi, Firenze
}

Survey of blood cultures methods in Italy

Key words: Microbiology, Survey, Diagnosis, Blood cultures, Management, Bacteraemia

\section{SUMMARY}

Sepsis is an important cause of morbidity and mortality; Blood cultures are the standard for identifying the responsible pathogen, bacteria or fungi. A number of factors influence the yield of blood culture, most of them concerning the microbiolist skill and the laboratory organization.

In order to collect information about the practices and procedures used for the detection of microrganisms in blood cultures in the italian laboratory, a questionnaire was sent to all the 2000 members of the Italian Association of Clinical Microbiology. Responses were received from 110 laboratories, located from all over the country (2.028.58I hospital admission). The results presented hereby concern specimen collection, culture techniques, rapid identification and susceptibility testing. In summary, most laboratories use automated systems (83.6\%), the lenght of incubation was 7 days in two out of three laboratories, although it is common to extend the incubation period when brucellosis (83 lab), endocarditis (47 lab), systemic mycosis (27 lab) is suspected. A wide variety of media are employed for subcultures. All laboratories examine the bottles at least once a day, while only 32 of 95 (33.7\%) laboratories processe the positive blood cultures on holiday. Communication between clinicians and microbiologist include: distribution of specimen collection guidelines by 93 ( $84.5 \%$ ) laboratories, availability of patients' clinical situation in 35 (32.4\%) laboratories, and adding to report the suggestion of potentialy contaminated culture (i.e."a positive results does not necessarly indicate bacteremias") in 3I (28.4\%). Only laboratories perform direct, tests $18.6 \%$ antimicrobial susceptibility test, and $9.3 \%$ perform rapid direct identification.

\section{INTRODUZIONE}

La sepsi costituisce una importante causa di morbilità, gravata da alta mortalità nelle forme severe $o$ associate a shock. Con l'emocoltura è possibile in molti casi confermare la diagnosi, identificare l'agente eziologico e definirne il pattern di sensibilità agli antibiotici. Il microbiologo è quindi coinvolto nella gestione di questi pazienti e deve assicurare l'attendibilità dei risultati, attraverso la scelta di metodi che assicurino il massimo di sensibilità e di specificità, e la loro disponibilità in tempi stretti, con il ricorso a tecniche rapide o preliminari e la tempestiva comunicazione dei risultati.

In letteratura sono disponibili numerose ed autorevoli indicazioni per la corretta esecuzione dell'emocoltura ed evidenziati gli aspetti consolidati e i momenti critici, cui il microbiologo dovrebbe far riferimento per assicurare l'ottimizzazione dei propri risultati $(10,16,18,29,33)$, ma anche $\mathrm{i}$ punti controversi su cui non c'è accordo unanime o mancano prove di documentata efficacia.

La raccolta di dati sulle modalità di esecuzione dell'emocoltura in Italia consente di delineare un quadro dell'esistente e quindi il confronto con le più aggiornate conoscenze, con la realtà di altri
Paesi, con le realtà locali.

Gli obiettivi dell'indagine possono così essere sintetizzati:

- conoscere le prassi diagnostiche ed operative della gestione e dell'emocoltura nei laboratori italiani,

- offrire elementi di riflessione e confronto a tutti i microbiologi (uno strumento per il miglioramento continuo della qualità è dato dal confronto con altre realtà, o benchmarking)

- disporre di riferimenti per la programmazione di iniziative di aggiornamento, mirate sulla base delle prassi esistenti.

\section{MATERIALI E METODI}

Nella primavera del 2002, per iniziativa congiunta del Comitato di Studio per la Batteriologia dell'Associazione Microbiologi Clinici Italiani (AMCLI) e dell'Associazione per la Prevenzione e lo Studio delle Infezioni (APSI), è stata avviata una indagine conoscitiva - relativa all'anno 2001 - sulle tecniche e le prassi dell'emocoltura per la diagnosi di batteriemie e di sepsi. Per semplicità, l'indagine è stata limitata alle emocolture effettuate con sistemi in brodo per la coltura di batteri 
e miceti, escludendo la ricerca di Micobatteri o di altri microrganismi particolari (quali, ad esempio, Legionella spp, Leptospira spp o Borrelia spp).

A tal fine è stato predisposto un apposito questionario che è stato distribuito, attraverso "Notizie AMCLI" periodico mensile dell'AMCLI, agli oltre 2000 colleghi iscritti all'Associazione, con l'invito alla compilazione da parte del Responsabile del laboratorio o Dirigente dallo stesso indicato.

Il questionario era diretto ad acquisire informazioni su: denominazione e caratteristiche del laboratorio, rapporti con i medici curanti, procedure diagnostiche utilizzate per le indagini microbiologiche sulle emocolture (con esclusione delle indagini per micobatteri e dei metodi non in brodo), carico di lavoro, rapporti con i medici curanti, gestione dei risultati. I risultati sono stati elaborati dopo inserimento in un foglio elettronico (Excel) ed in parte con Access. Prima dell'elaborazione i risultati sono stati verificati incrociando alcune risposte per verificarne la congruità $(\mathrm{ad}$ esempio, posti letto $v s$ numero di ricoveri $v s$ giornate di degenza). Per altri dati, mancanti o non comprensibili o apparentemente errati, si è provveduto a contattare il responsabile del Laboratorio per raccogliere o verificare l'informazione.

\section{RISULTATI}

Sono stati restituiti 120 questionari, di cui 110 risultati elaborabili (in 7 casi il laboratorio dichiara di non effettuare emocolture; in tre casi sono stati restituiti due questionario per una stessa istituzione).

I risultati sono riportati di seguito: nella lettura occorre tenere presente che non sempre e non tutti i punti del questionario sono stati compilati (per disattenzione, ma anche a non disponibilità del dato o a risultato negativo) e quindi non sempre $\mathrm{i}$ totali corrispondono al totale dei laboratori.

\section{Caratteristiche dei laboratori}

La struttura di appartenenza del laboratorio (lab.) è per 91 l'Ospedale, per 8 l'Università, per 7 un Istituto scientifico (IRCCS), per 4 una Case di cura privata, in un caso un Istituto geriatrico (tabella 1). I laboratori sono distribuiti, anche se in modo disomogeneo, in tutte le regioni italiane, con alto numero in Lombardia (23), Piemonte (14), Veneto (13), Campania (10), solo in parte giustificato dalla numerosa popolazione residente in queste regioni (tabella 2). Molto variabili anche li dimensioni delle strutture di ricovero che afferiscono ai laboratori: il numero di medio di posti letto (p.1.) è di 543 per struttura (range 68 -3000), con una distribuzione che vede 21 Ospedali con meno di 200 p.1., 35 con p.l. compresi tra i 200 e i 400, 24 con p.l. compresi tra i 401 e i 700, 16 con
701- 1000 p.l. e 14 con più di 1000 p.l. (tabella 3 ). Alcuni Colleghi specificano che l'attività del loro laboratorio è estesa a più presidi ospedalieri.

Nell'insieme i 110 laboratori servono 54.395 posti letto, per un totale di 2.028.581 ricoveri e 14.364.253 di giornate di degenza nel corso del 2001.

Tabella I. Sede del Laboratorio

\begin{tabular}{lll}
\hline \multirow{2}{*}{ Posti letto } & \multicolumn{2}{c}{ laboratori } \\
\cline { 2 - 3 } Ospedale & n. & $\%$ \\
\hline Università & 9 | & 82,7 \\
\hline Istituto scientifico (IRCCS) & 8 & 7,3 \\
\hline Casa di cura privata & 6 & 5,5 \\
\hline Istituto geriatrico & 4 & 3,6 \\
\hline
\end{tabular}

Tabella 2. Distribuzione geografica dei laboratori

\begin{tabular}{ll}
\hline Regione & n. laboratori \\
\hline Abruzzo & 3 \\
\hline Basilicata & 1 \\
\hline Calabria & 2 \\
\hline Campania & 10 \\
\hline Emilia Romagna & 5 \\
\hline Friuli Venezia Giulia & 3 \\
\hline Lazio & 7 \\
\hline Liguria & 3 \\
\hline Lombardia & 23 \\
\hline Marche & 2 \\
\hline Molise & 1 \\
\hline Piemonte & 4 \\
\hline Puglia & 6 \\
\hline Sardegna & 3 \\
\hline Sicilia & 3 \\
\hline Toscana & 5 \\
\hline Trentino Alto Adige & 4 \\
\hline Umbria & 2 \\
\hline Veneto & 13 \\
\hline
\end{tabular}

Tabella 3. Distribuzione degli Ospedali, sede dei laboratori, per numero di posti letto

\begin{tabular}{lll}
\hline \multirow{2}{*}{ Posti letto } & \multicolumn{2}{c}{ laboratori } \\
\cline { 2 - 3 }$<200$ & n. & $\%$ \\
\hline $200-400$ & 21 & $19, I$ \\
\hline $401-700$ & 35 & $3 I, 8$ \\
\hline $701-1000$ & 24 & 21,8 \\
\hline$>1000$ & 16 & 14,5 \\
\hline
\end{tabular}

\section{Sistemi utilizzati}

La grande maggioranza dei laboratori si avvale di strumenti che verificano periodicamente $o$ in continuo la rilevazione dei segni crescita nel brodo (92 lab. pari a 83,6\%): 58 con BACTEC (Becton Dickinson), 23 lab. con BacT/ALERT (bioMérieux), 11 con VITAL (bioMérieux); 4 lab. dichiarano di usare sia BACTEC che BacT/ALERT. Solo 18 laboratori (16,4\%) procedono alla lettura visiva della crescita: 8 utilizzando un sistema bifasioco (Hemoline Performance Duo, bioMérieux), 5 il sistema a flacone unico, 
bi, agar cioccolato, terreno selettivo per Gram negativi, terreno selettivo per Pseudomonas, terreno selettivo per salmonella, terreno selettivo per Gram positivi, terreno selettivo per Enterococchi, terreno selettivo per Stafilococchi, terreno selettivo per miceti). Per le sottocolture da flaconi aerobi si contano, su 99 lab. che forniscono l'informazione ben 45 diverse combinazioni dei terreni appena citati.

La tabella 9 riporta la frequenza di utilizzo dei vari terreni, per le sottocolture da flaconi aerobi, in 99 lab.: l'agar cioccolato è utilizzato da 83 lab. $(83,8 \%)$, l'agar sangue da 95 lab. (96\%) - altri due lab. utilizzano il solo agar sangue in anaerobiosi -, un terreno selettivo per Gram negativi dal $78,8 \%$ (cui un $10 \%$ dei lab. associa un terreno selettivo per Pseudomonas spp. ed un altro $10 \%$ anche il terreno selettivo per Salmonella spp.), un terreno selettivo per Gram positivi da $37,4 \%$ dei lab. (associato nel 26,3\% ad un terreno selettivo per stafilococchi e nel $14,1 \%$ ad un terreno selettivo per Enterococchi); infine, l'agar Sabouraud è utilizzato dal $68,7 \%$ dei lab. Infine, 79 lab. (79,8\%) associa l'agar sangue all'agar cioccolato.

Tabella 9. Terreni utilizzati per sottocolture da flaconi aerobi (rispondono $99 \mathrm{lab}$ )

\begin{tabular}{lll}
\hline TERRENO & \multicolumn{2}{c}{ Lab } \\
\cline { 2 - 3 } & N. & $\%$ \\
\hline Agar Cioccolato & 83 & 83,8 \\
\hline Agar Sangue & 95 & 96 \\
\hline Agar sangue per anaerobi & 9 & 9,1 \\
\hline Agar sangue per anaerobi, selettivo & 3 & 3,0 \\
\hline Terreno selettivo per Cocchi Gram positivi & 37 & 37,4 \\
\hline Terreno selettivo per Stafilococchi & 56 & 56,6 \\
\hline Terreno selettivo per Enterococchi & 19 & 19,2 \\
\hline Terreno selettivo per Gram negativi & 78 & 78,8 \\
\hline Terreno selettivo per Pseudomonas & 10 & 10,1 \\
\hline Terreno per Salmonella & 10 & 10,1 \\
\hline Terreno per miceti (tipo Sabouraud) & 68 & 68,7 \\
\hline Altri terreni & 6 & 6,1 \\
\hline
\end{tabular}

\section{Utilizzo di metodi che consentono di anticipare} i risultati

Il questionario indagava anche alcuni aspetti del-
Tabella 7. Distribuzione dei lab. per la durata dellincubazione (giorni) in uso nella routine

\begin{tabular}{cllllll}
\hline \multirow{2}{*}{ GG di incubazione } & \multicolumn{2}{c}{ aerobi } & \multicolumn{2}{c}{ Anaerobi } & \multicolumn{2}{c}{ miceti } \\
\cline { 2 - 8 } & n. & $\%$ & n. & $\%$ & n. & $\%$ \\
\hline 3 & $\mathrm{I}$ & 1,0 & $\mathrm{I}$ & 0,9 & $\mathrm{I}$ & 2,4 \\
\hline 5 & 10 & 9,5 & 10 & 9,2 & $\mathrm{I}$ & 2,4 \\
\hline 6 & 5 & 4,8 & 5 & 4,6 & & \\
\hline 7 & 74 & 70,5 & 78 & 71,6 & 16 & 38,1 \\
\hline 8 & 3 & 2,9 & 3 & 2,8 & & \\
\hline 10 & 9 & 8,6 & 10 & 9,2 & 7 & 16,7 \\
\hline 14 & $\mathrm{I}$ & 1,0 & $\mathrm{I}$ & 0,9 & 13 & 31,0 \\
\hline 15 & $\mathrm{I}$ & 1,0 & $\mathrm{I}$ & 0,9 & 3 & 7,1 \\
\hline 20 & $\mathrm{I}$ & 1,0 & & & & \\
\hline 45 & & & & & $\mathrm{I}$ & 2,4 \\
\hline tot. Risposte & 105 & & 109 & & 42 & \\
\hline
\end{tabular}

Tabella 8. Distribuzione dei lab. in base al motivo di prolungamento dellincubazione ed alla durata della stessa

\begin{tabular}{|c|c|c|c|c|}
\hline \multirow{2}{*}{ GG di incubazione } & \multicolumn{4}{|c|}{ Maggior durata dell'incubazione per: } \\
\hline & Brucellosi & Endocardite & AIDS & Micosi \\
\hline 7 & & & $\mathrm{I}$ & 1 \\
\hline 8 & & & & 1 \\
\hline 10 & 5 & 7 & 2 & 6 \\
\hline 12 & $\mathrm{I}$ & 1 & & \\
\hline 14 & 16 & 11 & 2 & 8 \\
\hline 15 & 16 & 11 & & 3 \\
\hline 18 & & & 1 & \\
\hline 20 & 17 & 7 & & 1 \\
\hline 21 & 13 & 7 & 1 & 5 \\
\hline 25 & 2 & & & \\
\hline 28 & 6 & 2 & & 1 \\
\hline 30 & 3 & $\mathrm{I}$ & & 1 \\
\hline 40 & $\mathrm{I}$ & & & \\
\hline tot & 80 & 47 & 7 & 27 \\
\hline
\end{tabular}

l'esame dei brodi di emocoltura, particolarmente critici in ordine alla tempestività della risposta e/o alla possibilità di anticipare informazioni parziali o preliminari al clinico:

- la frequenza di osservazione dei brodi per rilevazione dei segni di crescita, durante la settimana e nel week end,

- l'effettuazione dell'esame microscopico sui brodi con segni di crescita,

- l'effetttuazione di test "diretti", effettuati utilizzando direttamente la brodocoltura, senza attendere la crescita di colonie sui terreni solidi.

I flaconi vengono esaminati almeno una volta al giorno da lunedì a sabato in tutti i lab., ma in molti lab. anche due volte al giorno. Diversa la situazione nel fine settimana: non esaminano $\mathrm{i}$ flaconi delle emocolture 4 su 101 lab. (4\%) il sabato e 63 su 95 lab. $(66,3 \%)$ la domenica. Tra i 32 laboratori che esaminano i campioni nei giorni festivi ci sono laboratori di strutture di varie dimensioni; la tabella 10 riporta il numero e la frequenza dei laboratori che esaminano i campioni nei giorni festivi nell'ambito di Laboratori di 
ospedali di analoghe dimensioni.

Tabella 10. Laboratori che esaminano i campioni di emocoltura: numero e frequenza nell'ambito di strutture di analoghe dimensioni

\begin{tabular}{lccc}
\hline \multirow{2}{*}{ Posti letto } & \multicolumn{3}{c}{ laboratori } \\
\cline { 2 - 4 } & Totale & Esaminano i campioni nei giorni festivi \\
\hline$<200$ & 21 & n. & $\%$ \\
\hline $200-400$ & 35 & 6 & 28,6 \\
\hline $401-700$ & 24 & 12 & 34,3 \\
\hline $701-1000$ & 16 & 5 & 20,8 \\
\hline$>1000$ & 14 & 4 & 25,0 \\
\hline
\end{tabular}

L'esame microscopico (forniscono questa informazione 108 lab.) viene effettuato routinariamente da 93 lab. (86,1\% dei lab.), generalmente dopo colorazione di Gram (6 lab. usano arancio di acridina, 1 lab, blu di metilene).

L'identificazione diretta, sembra non far parte del bagaglio colturale, o almeno delle possibilità operative dei microbiologi italiani: solo 10 su 107 $(9,3 \%)$ dichiarano di procedere all'identificazione diretta sul brodo. Il dato deve però essere criticamente perché è possibile che qualche identificazione diretta venga effettuata ancorché non in modo sistematico e su tutti gli isolati.

I dati sull'antibiogramma diretto sembrano però confermare la scarsa propensione dei microbiologi italiani all'esecuzione di test diretti: solo il $18,6 \%$ dei lab. (19 su 102) effettua l'antibigramma diretto. I metodi utilizzati sono il Kirby Bauer (12 lab.), lo Sceptor (2), Kirby Bauer e/o Sensititre (1), Kirby Bauer e/o ATB (1), Microscan (1). La distribuzione dei laboratori che effettuano l'antibiogramma diretto, per dimensione della struttura di appartenenza, è riportata nella tabella 11.

Tabella I I. Laboratori che effettuano test "diretti” di sensibilità: numero e frequenza nell'ambito di strutture di analoghe dimensioni

\begin{tabular}{llcl}
\hline Posti letto & \multicolumn{3}{c}{ laboratori } \\
\cline { 2 - 4 } & Totale & \multicolumn{2}{c}{ Effettuano test “diretti” di sensibilità } \\
\cline { 2 - 4 } & & $\mathrm{n}$. & $\%$ \\
\hline$<200$ & 21 & 4 & 19,0 \\
\hline $200-400$ & 35 & 2 & 5,7 \\
\hline $401-700$ & 24 & 5 & 20,8 \\
\hline $701-1000$ & 16 & 1 & 6,3 \\
\hline$>1000$ & 14 & 7 & 50,0 \\
\hline
\end{tabular}

\section{Rapporti con i medici curanti}

Il questionario chiedeva notizie sullo scambio di informazioni con i medici curanti: dal microbiologo al clinico per la fase pre-analitica (suggerimenti per la corretta esecuzione del prelievo) e nell'interpretazione del referto (segnalazione di possibili contaminanti), dal clinico al microbiologo (informazioni clinico anamnestiche utili ad ottimizzare la fase analitica (tabella 12).
Tabella I2. Collaborazione con i medici curanti

\begin{tabular}{llll}
\hline & NO & SI & n.r. \\
\hline Indicazioni scritte alle UO per prelievo & 16 & 93 & I \\
\hline Informazioni cliniche sul Modulo di richiesta & 35 & 73 & 2 \\
\hline Indicazione di sospetta contaminante & 31 & 78 & I \\
\hline n.r. non risponde & & &
\end{tabular}

Alla domanda "avete fornito ai reparti indicazioni scritte sulle modalità di prelievo?" rispondono 109 laboratori: sì 93 lab. (85.3\%), no 16 lab. (14.7\%). 44 colleghi ci hanno inviato copia del modulo con le indicazioni per la raccolta; la tabella 13 ne riferisce i contenuti.

Alla domanda "il modulo di prevede la trasmissione al laboratorio di notizie cliniche?" rispondono 108 laboratori: sì 73 (7.5\%), no 35 (24\%). Il contenuto dell'informazione è stato analizzato, secondo il punto di vista degli AA, rispetto alle informazioni utili per l'ottimizzazione della diagnosi microbiologica (tabella 14).

Infine, veniva chiesto "indicate sul referto se il germe isolato è un probabile contaminante?": lo segnalano 78 laboratori $(71,6 \%)$, non lo segnalano $31(28,4 \%)$, non risponde un laboratorio.

Tabella 13. Analisi dei moduli, prodotti e distribuiti dal lab, con istruzioni e/o indicazioni sulle modalità di prelievo per l'emocoltura Tipo di informazione Laboratori che la forniscono

\begin{tabular}{lll}
\hline Premessa clinica & n. & $\%$ \\
\hline Modalità di preparazione cute * & 5 & $I I, 4$ \\
\hline Numero e tempi di prelievo & 38 & 86,4 \\
\hline Quantità di sangue & 23 & 52,3 \\
\hline Tipo di flaconi & 31 & 70,5 \\
\hline Istruzioni per portatori CVC & 24 & 54,5 \\
\hline Modalità di comunicazione dei referti & 4 & 13,6 \\
\hline Modifiche dell'iter diagnostico sulla base & & \\
di specifiche situazioni cliniche & 12 & 27,3 \\
\hline * in alcuni casi le indicazioni risultano poco dettagliate &
\end{tabular}

Tabella 14. Analisi dei moduli di richiesta delle indagini microbiologiche rispetto alle informazioni richieste al clinico per ottimizzare l'iter dell'indagine microbiologica

Livello di completezza Distribuzione dei Laboratori \begin{tabular}{lll} 
dell'informazione & n. & $\%$ \\
\hline Esauriente per l'esame & II & 25,6
\end{tabular}

\begin{tabular}{lll}
\hline Esauriente per l'esame & I I & 25,6 \\
\hline Poco esaurienti per l'esame & II & 25,6
\end{tabular}

Equivalenti a nessuna notizia

$21 \quad 48,8$

\section{DISCUSSIONE}

L'emocoltura è fra gli esami di laboratorio che vengono richiesti in quantità relativamente scarsa rispetto alla reale importanza diagnostica. Per esemplificare vengono riportate qui di seguito le principali situazioni cliniche in cui è importante eseguire il test e la \% attesa di positività (3): Endocarditi ed infezioni endovascolari (85 95\%), Epiglottite acuta $(80-90 \%)$, Polmonite batterica $(5-30 \%)$, Pielonefrite ascendente $(30-$ $50 \%)$, Osteomielite ematogena $(30-50 \%)$, 
Meningite batterica $(50-80 \%)$, Ascessi endoaddominali e Febbre di origine sconosciuta (varia). L'importanza di effettuazione dell'emocoltura è ribadita dall'autorevole Linea Guida, prodotta della Society od Critical Medicine e della Infectious Disease Society of America, riferita in verità ai pazienti delle Terapie intensive, che afferma: "Because the information provided by a positive blood culture can have such important prognostic and therapeutic implications, blood cultures should be performed for patients with new fever, even when the clinical findings do not strongly suggest a noninfectious cause (30)". Ed aggiungiamo noi, deve essere effettuata in laboratorio secondo le migliori prassi di laboratorio.

L'emocoltura consente di confermare il sospetto clinico di sepsi, di accertare l'agente eziologico in causa e studiarne la sensibilità in vitro agli antibiotici. Il risultato dell'indagine, e il suo impatto sulla cura del paziente, sono condizionati da molteplici fattori fra cui principalmente il volume del campione, il momento del prelievo, l'intervallo ed il numero dei prelievi, l'accuratezza del prelievo (metodo di disinfezione della cute), le caratteristiche del mezzo di coltura, la durata dell'incubazione, la capacità del sistema analitico di evidenziare lo sviluppo batterico, l'interpretazione del risultato (patogeni $v s$ contaminanti).

Conoscere la situazione nel nostro Paese può fornire utili informazioni: all'Autorità sanitaria ed alle Associazioni scientifiche per programmare iniziative di formazione e formulare linee guida, ai singoli microbiologi per confrontare le proprie prassi con quelle di altri colleghi, ai clinici per ottimizzare la richiesta dell'esame e il miglior uso dei risultati.

L'indagine da noi effettuata ha raccolto informazioni sull'attività di 110 laboratori, che possono essere considerati un buon campione della realtà italiana in quanto esaminano circa un terzo delle emocolture italiane (secondo il rapporto di Databank, dicembre 2001) e sono distribuiti su tutto il Paese; un bias potrebbe essere costituito dalla modalità di arruolamento (gli iscritti alla Associazione professionale potrebbero essere più sensibili sulle tematiche) e dalla selezione dei rispondenti (ancora una volta i più interessati e motivati).

Riteniamo poi utile confrontare i dati relativo al 2001 con quelli raccolti in precedenti indagini italiane, per verificare gli eventuali cambiamenti, i trend e i problemi irrisolti ni con dati USA (2I)
(11) e di altri Paesi $(21,22)$.

Dall'analisi delle risposte emergono alcuni aspetti che meritano una riflessione e la formulazione di proposte indirizzate a migliorare l'attività diagnostica microbiologica.

Diversi i documenti autorevoli cui attingere - a cui anche noi faremo riferimento - per la corretta esecuzione dell'emocoltura $(10,18,29,33)$, anche se alcuni aspetti sono ancora controversi o mancano prove di efficacia.

\section{La richiesta di emocoltura}

Un punto critico è certamente rappresentato dalla decisione di effettuare l'emocoltura. Pur non costituendo l'obiettivo primario dell'indagine, i dati raccolti forniscono informazioni - o almeno indicazioni - sulle abitudini di ricorso all'emocoltura negli ospedali italiani. I dati raccolti evidenziano complessivamente uno scarsa abitudine all'esecuzione dell'emocoltura negli ospedali italiani (tabella 15), almeno rispetto agli USA (21), anche se con spiccate diversità nelle diverse realtà come indicato dall'ampio range, pur tenendo conto delle dimensioni degli Ospedali in cui opera il laboratorio: da 0,03 a 13,4 set di emocoltura per p.1., da 3,7 a 1183 set per 1.000 giornate di degenza, da $0,1 \mathrm{a}-78,2$ set per 1.000 ricoveri. L'aspetto positivo, che merita di essere sottolineato è l'incremento osservato rispetto alla precedente rilevazione, effettuata in ambito AMCLI, con analoga metodologia (tabella 16), anche se non sullo stesso campione.

\section{Aspetti metodologici}

a) Volume di sangue, numero e tempi di prelievo. Esiste una relazione diretta fra volume di sangue prelevato, indipendentemente dal numero di flaconi inoculati, e positività $(10,17,24,30)$. La carica batterica, sia nelle forme di batteriemia intermittente che in quelle continue per infezione endovascolare è infatti molto bassa, dell'ordine di $1-10 \mathrm{cfu} / \mathrm{mL}$ (36). In letteratura si raccomanda per l'adulto il prelievo di almeno $20-30 \mathrm{ml}$ di sangue $(10,18,29,30)$; in età pediatrica sono, invece, sufficienti volumi minori poiché nei bam-

Tabella 15. Distribuzione (\%) dei lab. per n. di flaconi esaminati: confronto dei dati italia-

\begin{tabular}{lllll}
\hline & \multicolumn{4}{c}{ Numero di flaconi esaminati } \\
\cline { 2 - 5 } & $<1500$ & $\mathrm{I} 50 \mathrm{I}-3500$ & $350 \mathrm{I}-8000$ & $>8000$ \\
\hline Italia, I09 Ospedali (200I) & 39,4 & 23,2 & 22,2 & 15,2 \\
\hline USA, 258 Ospedali (1986) & 17,1 & 23,3 & 37,2 & 22,5 \\
\hline
\end{tabular}

Tabella I6. Distribuzione (\%) dei lab. per n. di flaconi esaminati: confronto dei dati dell'indagine con analoga indagine effettuata nel 1988 [I I]

\begin{tabular}{lllllll}
\hline \multicolumn{7}{c}{ Numero di flaconi esaminati } \\
\hline & $<500$ & $500-$ & $1.00 I-$ & $2.00 I-$ & $5.00 I-$ & $>10.000$ \\
& & 1.000 & 2.000 & 5.000 & 10.000 & 0 \\
\hline Italia, 109 Ospedali (200I) & $I 7, I$ & $I 7, I$ & 10,5 & 30,5 & $I I, 4$ & 13,3 \\
\hline Italia, I45 Ospedali (1988) & 50,3 & $22, I$ & 12,4 & 11,0 & 2,8 & $I, 4$ \\
\hline
\end{tabular}


bini la batteriemia presenta una carica microbica più elevata (29).

I nostri dati sono riferiti al numero di flaconi, fornendo una indicazione solo indiretta dei volumi prelevati (i sistemi più diffusi prevedono l'inoculo di $5-10 \mathrm{ml}$ per flacone); un $40 \%$ di laboratori dichiara di rilevare meno di 4 flaconi per paziente (tabella 6) che potrebbero non assicurare un volume sufficiente di sangue.

Anche sul numero di prelievi (set) si trovano indicazioni non univoche in letteratura, spesso con riferimenti e indicazioni diverse in base alla patologia sottostante (18). La totalità degli AA raccomanda il prelievo di 2 o 3 set di emocoltura. Ulteriori emocolture, nei giorni successivi, potrebbero risultare utili nei pazienti in terapia antibiotica, con febbre di origine oscura (ad esempio, ascessi occulti, tifo, brucellosi) o con sospetta endocardite subacuta $(10,18)$. Il vantaggio di più prelievi è ben illustrato da Lamy et al. in una recente lettera al CID (19): supponendo una frequenza di contaminazione del $25 \%$ per set si avrebbe una probabilità del $58 \%$ di isolare un contaminante in almeno un set; per contro la probabilità di trovare lo stesso contaminante in due set scende al $15,6 \%$ e in tre set all' $1,6 \%$.

Circa i tempi dei prelievi le indicazioni fornite dalla letteratura parlano di prelievi distanziati di $10-20$ ': non esistono però prove a supporto (25).

\section{b) Tempo di incubazione}

Per quanto riguarda i sistemi manuali la durata dell'incubazione consigliata, salvo le condizioni cliniche e microbiologiche che prevedono un prolungamento, è di sette giorni. Per quanto riguarda i sistemi assistiti da strumentazione la maggior parte degli autori suggerisce tempi di incubazione di 5-7 giorni, sempre escludendo i casi di necessario prolungamento. I dati raccolti appaiono pienamente in linea con le indicazioni della letteratura $(10,18,29)$. Nella esperienza di uno degli Autori dopo 5 giorni si ha crescita del 98,9\% dei patogeni, contro il $99,8 \%$ delle crescite dopo 7 giorni (27).

Dall'indagine risulta che molti laboratori prolungano l'incubazione per particolari situazioni cliniche (sospetta brucellosi, endocardite, AIDS, micosi sistemiche). È però il caso di sottolineare che su questo aspetto, pur sottolineato in tutta la letteratura, manca un consensus su quali siano le situazioni in cui prolungare l'incubazione e quanto a lungo.

\section{c) Controllo della positivizzazione}

La grande maggioranza dei laboratori italiani utilizza strumenti che provvedono alla rilevazione in continuo dei segni di crescita. Chi utilizza sistemi manuali esamina almeno una volta al giorno le emocolture per rilevare i segni di crescita; 3 su 17 esaminano i flaconi due o più volte al giorno.

Un punto dolente è la mancata effettuazione dei test microbiologici (esame microscopico, sottocolture e test "diretti"), nei giorni festivi da parte di 63 lab su 93 (66,3\%). Curioso che il dato non sia correlabile con le dimensioni degli Ospedali serviti dal laboratorio; nei grandi Ospedali sono ricoverati pazienti a maggior rischio di sepsi severe e la frequenza di riscontro di emocolture positive è certamente più alta. La decisone dell'apertura, almeno parziale, della microbiologia non può essere decisa autonomamente dai microbiologi; ma certamente il microbiologo dovrebbe farsi carico di sollecitarla, anche se, in tempi di grande attenzione alle spese correnti, potrebbe risultare difficile trovare interlocutori sensibili.

Nel caso in cui, per carenza di risorse, questo non sia possibile si raccomanda di accordarsi con i reparti di degenza in modo che, in caso di gravi condizioni di pazienti con emocolture in corso, il clinico possa ricorrere al servizio di reperibilità del laboratorio.

\section{d) Esame microscopico}

Da ogni flacone positivo deve essere effettuato tempestivamente un esame microscopico (Gram e/o arancio di acridina) per confermare la positività e osservare la morfologia presenza di miceti o batteri, e per questi ultimi, i caratteri tintoriali $(10,18,29,37)$. Tale prassi risulta ampiamente diffusa, con una quota però non piccola di laboratori (15 su 108, pari al 13,9\%) che dichiarano di non effettuare l'esame microscopico: questi ultimi sono variamente distribuiti nel Paese e operano in ospedali di varie dimensioni (da 90 a 1130 p.1.). Largamente utilizzata la colorazione di Gram (80 lab., pari all'86\%); 6 lab. (6,5\%) utilizzano la colorazione di arancio di acridina: manca l'informazione sulle caratteristiche tintoriali, ma offre una maggior sensibilità (utili in caso di batteriemie sostenute germi che si colorano poco o con difficoltà, quali Brucella spp o Campylobacter spp).

Il referto deve essere il più descrittivo possibile $(10,18)$. Se la lettura è fatta da un microbiologo esperto è preferibile specificare "cocchi Gram positivi a grappolo: probabile stafilococco" piuttosto che "cocchi Gram positivi" (29). Il referto va comunicato tempestivamente, per telefono o fax, al medico curante. Il test serve anche come guida all'esecuzione di eventuale identificazione ed antibiogramma "diretti".

Attenzione però: deve essere chiarito al medico curante che microscopicamente non possiamo distinguere lo stafilococco aureo dagli altri stafilococchi per cui occorre molta prudenza nell'interpretazione del risultato perché potrebbe essere un inquinante! 


\section{e) Sottocoltura su terreni solidi}

In letteratura viene raccomandata la sottocoltura su: Agar sangue (in aerobiosi), agar sangue per anaerobi (in anaerobiosi). Molti AA $(18,29)$ raccomandano anche la semina su agar cioccolato (in $\mathrm{CO}_{2}$ ), su un terreno selettivo-differenziale per Gram negativi e su un terreno selettivo per Gram positivi (aggiunti di routine, o sulla base del Gram, risultano particolarmente utili in caso di batteriemie polimicrobiche).

La realtà italiana appare estremamente variegata. Considerando le sole sottocolture da flaconi aerobi, rileviamo una diffusa abitudine all'uso di agar sangue, con l'eccezione di quattro lab (due seminano però sempre sa agar sangue per anaerobi, gli altri due sono piccoli laboratori), un uso solo nell' $83,8 \%$ dell'agar cioccolato (necessario invece per la crescita di germi esigenti, non raramente in causa nelle batteriemie), un ampio uso di terreni selettivi per Gram negativi e Gram positivi, l'uso ancora di terreni selettivi, diversamente da quanto indicato in letteratura, per Salmonella spp (10\% dei lab), Pseudomonas spp (10\%) e Miceti $(68,7 \%)$. Salvo specifiche situazioni epidemiologiche l'uso nella routine di questi ultimi terreni non sembra giustificato, soprattutto alla luce dei maggiori costi.

\section{f) Test "diretti" per l'identificazione e l'antibio- gramma}

È bene dire subito che non ci sono procedure approvate né standardizzate; ciò nonostante molti Autori ne consigliano l'esecuzione $(16,18)$. L'uso dei test diretti viene lasciato alla responsabilità ed esperienza personale tenendo presente che risultati scorretti possono fuorviare l'indirizzo terapeutico e risultare dannosi: quindi massima prudenza nell'interpretazione del risultato da comunicare al clinico.

Esistono però numerosi lavori in letteratura che propongono modalità affidabili di identificazione "diretta" $(1,2,6,8,14,23,32,33,34,38)$; un algoritmo particolarmente dettagliato è riportato sul testo di Isenberg (18). L'esperienza anche di colleghi italiani $(4,11)$, ha dimostrato che gli errori negli antibiogrammi diretti sono quasi solo "minor error".

Anticipare di 24 ore il risultato, almeno dell'antibiogramma, dovrebbe avere una positiva ricaduta clinica (mancano peraltro lavori in letteratura che lo documentino), tanto più importante in una patologia, la sepsi, che può avere una rapida evoluzione clinica e, nelle forme severe o con shock, una alta mortalità, compresa tra il 20 e il $50 \%(9$, 20,33).

In altri Paesi molti lab. effettuano test diretti: dall'indagine americana, già citata (21), risulta che il $31 \%$ dei laboratori effettua test diretti per l'iden- tificazione e il $75 \%$ test diretti per l'antibiogramma. In Italia test diretti sono effettuati per identificazione ed antibiogramma rispettivamente dal $9.3 \%$ e dal $17,9 \%$; poco è cambiato, in peggio, dalla rilevazione del 1988 quando questi test venivano effettuati rispettivamente dall' $8.7 \%$ e $25 \%$ dei laboratori. I test diretti risultano effettuati soprattutto nei laboratori di grandi dimensioni (il $50 \%$ dei laboratori in strutture con più di 1000 posti letto).

\section{g) Collaborazione con $\boldsymbol{i}$ clinici}

L'utilità di una stretta collaborazione tra medico curante e microbiologo è stata spesso enfatizzata e risulta particolarmente importante nel caso dell'emocoltura: Tre gli aspetti considerati dal questionario.

La disponibilità per il microbiologo di informazioni cliniche sul paziente per decidere eventuale strategie alternative alla routine: è il caso del prolungamento dell'incubazione nel sospetto di particolari patologie. Tale prassi sembra essere ancora poco diffusa nel nostro Paese: solo due terzi dei laboratori (73 lab. su 108, pari a 67,6\%) riceve informazioni, che però solo in una metà dei casi possono essere utili per ottimizzare l'esame (tabelle 10 e 12).

Più diffusa invece (93 lab. su 109, pari all' $85,3 \%$ ) la prassi di produrre e distribuire all'utenza indicazioni per la fase preanalitica (tabelle 10 e 11), segno di una crescente sensibilità del microbiologo ad affrontare temi al di fuori dello stretto ambito analitico. Nell'indagine del 1988 era risultato che riceveva notizie cliniche il $62.5 \%$ dei lab., ed aveva prodotto e distribuito indicazioni scritte per la raccolta il $60 \%$.

Molti lab. (78 su 109, pari 71,6\%), di fronte all'isolamento di un probabile contaminate, ne danno indicazione sul referto; tale prassi appare in linea con le indicazioni della letteratura $(16,18)$, anche se l'interpretazione del risultato non è sempre agevole (40). Assai significativi i risultati di un'indagine effettuata in un Ospedale inglese (28): il 97\% dei medici ha detto di approvare l'inserimento nel referto di un commento del microbiologo (ed esempio: "probabile infezione" o "probabile contaminazione"). Anche se il giudizio definitivo spetta al curante, il microbiologo può portare un importante contributo rispetto al significato clinico del germe isolato, nell'ambito di una stretta collaborazione tra clinico e laboratorista $(7,31)$.

\section{CONCLUSIONI}

I dati raccolti indicano una sostanziale diffusa correttezza nell'esecuzione dell'emocoltura, con una buona adesione ai protocolli diagnostici prodotti dalle società scientifiche o agenzie sanitarie. 
É però anche vero che non esistono in letteratura indicazioni cogenti in molti casi, ma piuttosto indicazioni; di alcuni aspetti, ad esempio sui tempi e il numero dei prelievi, si trovano indicazioni non sempre concordanti; in altri casi mancano evidenze, come per la scelta o meno dell'uso di inibitori degli antibiotici nei brodi (resine o FAN).

Un aspetto critico, che l'indagine ha evidenziato, è la scarsa attenzione ai tempi di risposta (turn around time): mancata esecuzione in alcuni casi al momento della rilevazione della positività dell'emocoltura, scarsa diffusione dei test diretti, mancata effettuazione dei test microbiologici dei campioni nei giorni festivi. Su questo aspetto è auspicabile un forte impegno delle società scientifiche attraverso l'organizzazione di momenti formativi e la sensibilizzazione delle autorità sanitarie, ma anche dei microbiologi per attivare iniziative di scambio e di confronto con i colleghi clinici.

Infine, un grazie ai numerosi colleghi che hanno saputo e voluto trovare il tempo per raccogliere e comunicarci i loro dati.

\section{BIBLIOGRAFIA}

1. Applebaum PC, Schick SF, Kellogg JA. Evaluation of the four-hour Micro-ID technique for direct identification of oxidase-negative, gram-negative rods from blood cultures. J Clin Microbiol 1980; 12: 533-7.

2. Blazevic DJ, Trombley CM, Lund ME. Inoculation of API-20E from positive blood cultures. J Clin Microbiol 1976; 4: 522-3.

3. Braga A, Botta G. Aspetti metodologici ed interpretativi delle emocolture. L'Igiene Moderna 1990; 93: 35-49.

4. Calì AM, Gualdi P, Maffei R, Schinella M. Tra microbiologia tradizionale e tecniche molecolari: le possibilità per un laboratorio medico di ridurre il TAT e migliorare l'outcome nella diagnostica delle infezioni del sangue. Microbiol Medica 2002; 17: 96-7.

5. Callegaro A, Basaglia G, Mucignat G, Pascoli L, Tarabini G, Santini G. Uso di E-Test per il saggio di sensibilità diretto da emoculture. Microbiologia Medica 1995; 10: 100-1.

6. Chorny JA, Wilson ML. Rapid detection and identification of microorganisms from blood cultures. Clin Lab Med 1994; 14: 181-95.

7. Cunney RJ, Smith EG. The impact of laboratory reporting practice on antibiotic utilisation. International J Antimicrobial Agents 2000; 14: 13-9.

8. Davis TE, Fuller DD, Aeschleman EC. Rapid, direct identification of Staphylococcus aureus and Streptococcus pneumoniae from blood cultures using commercial immunologic kits and modified conventional tests. Diagn Microbiol Infect Dis 1992; 15: 295-300.

9. Doern GV, Vautour R, Gaudet M, Levy Bruce. Clinical Impact of Rapid In Vitro Susceptibility Testing and Bacterial Identification. J Clin Microbiol 1994; 32: 1757-62.

10. Dunne WM Jr, FS Nolte, ML Wilson. Cumitech 1B
Blood Cultures III. April 1997 American Society for Microbiology, Washington D.C. , USA

11. Goglio A, Marchiaro G, Notarnicola MA, Piacentini I, Scagnelli M. L'emocoltura nei laboratori italiani. L'igiene moderna 1990; 93: 143-59.

12. Goglio A, Marchiaro G. Affidabilità dell'antibiogramma diretto da emocolture. BML 1993;1: 41-8.

13. Goglio A. La sepsi: rilevanza clinica e problemi di diagnosi microbiologica con metodi rapidi non automatizzati. Emergenza e diagnosi rapida in microbiologia clinica, Monografia di Microbiologia Medica dell'Associazione Microbiologi Clinici Italiani, Milano, 1985.

14. Gordon LP, Damm MAS, Anderson JD. Rapid presumptive identification of streptococci directly from blood cultures by serologic tests and the L-pyrrolidonyl-b-naphthylamide reaction. J Clin Microbiol 1987; 25: 238-41.

15. Gosbell IB, Newton PJ, Sullivan EA. Survey of blood cultures from five community hospitals in south-western Sydney, Australia, 1993-1994. Aust NZ J Med 2000, Apr, 30(2): 286-7.

16. Health Protection Agency. Standard operative procedure. Investigation of blood cultures (for organisms other than Mycobacterium species). BSOP 37, Issued by Standards Units, Evaluations and Standards Laboratory, http://www.phls.co.uk/dir/hq/sops/bsoppdf/bsop37i3 .1.pdf. Issue date: 02.06 .03

17. Ilstrup DM, Washington JA. The importance of volume of blood cultured in the detection of bacteremia and fungemia. Diagn Microbiol Infect Dis 1983; 1: 107-10.

18. Isemberg HD. Clinical Microbiology Procedures Handbook. 1992. American Society for Microbiology, Washington D.C., USA

19. Johnson JR. Multiple Blood Cultures for Diagnosing Bacteremia. Clin Infect Dis 2003; 37: 738.

20. Kang CI, Kim SH, Kim HB, et al. Pseudomonas aeruginosa Bacteremia: Risk factor for mortality ad Influence of Delayed Receipt of Effective Antimicrobial Therapy on Clinical Outcome. Clin Infect Dis 2003; 37: 745-51.

21. Kehl KSC. Results of Survey of Blood culture Methods: part I Cl Microbiol Newsletter 1986; 8, 117-120

22. Kehl KSC. Results of Survey of Blood culture Methods: part II Cl Microbiol Newsletter 1986; 8, 127-32.

23. Knight RG, Shlaes DM. Rapid identification of Staphylococcus aureus and Streptococcus pneumoniae from blood cultures. J Clin Microbiol 1983; 17: 97-9.

24. Lamy B, Roy P, Carret G, Flandrois J-P, DelignetteMuller ML. What is the relevance of obtaining mulptiple bloo samples for cultures? A compehensive model to optimize the strategy for diagnosing bacteremia. Clin Infect Dis 2002; 35: 842-50.

25. Li J, Plorde JJ, Carlson LG. Effects of volume and periodicity on blood cultures. J Clin Microbiol 1994; 32: 2829-31.

26. Mermel LA, Maki DG. Detection of becteremia in adults: consequences of culturing an inadequate volume of blood. Ann Intern Med 1993; 119: 270-2.

27. Moioli F, Arosio M, Facheris MA, et al. Emocolture: valutazione dei tempi di crescita con il sistema Bact/Aler. Microbiologia Medica 2001; 16: 219 (O).

28. Morgan MS. Perceptions of a medical microbiology service: a survey of laboratory users. J Clin Path 1995; 48: 915-8. 
29. Murray PR, Baron EJ, Jorgensen JH, Pfaller MA, Yolken RH. Manual of Clinical Microbiology, $8^{\text {th }}$ ed., American Society for Microbiology, Washington D.C., USA.

30. O'Grady NP, Barie PS, Bartlett JG, et al. Practice Guidelines for evaluating new fever in critically ill adult patients. Clinical Infectious Diseases 1998; 26: 1042-59.

31. O'Hara CM, Weinstein MP, Miller JM. Manual and Automated Systems for Detection and Identification of Microrganisms. In: Murray PR, Baron EJ, Jorgensen JH, Pfaller MA, Yolken RH. Manual of Clinical Microbiology, $8^{\text {th }}$ ed., American Society for Microbiology., Washington D.C., USA.

32. Rappaport T, Sawyer KP, Nachamkin I. Evaluation of several commercial biochemical and immunologic methods for rapid identification of Gram-positive cocci directly from blood cultures. J Clin Microbiol 1988; 28: 1335-8.

33. Reimer LG, ML Wilson, Weinstein MP. Update on detection of bacteremia and fungemia. Clin Microbiol Rev 1997, 10: 444-65.

34. Schifman RB, Ryan KJ. Rapid automated identification of Gram-negative bacilli from blood cultures with the Auto Microbic system. J Clin Microbiol 1982; 15: 260-4.

35. Sewell DL, MacLowry JD. Laboratory management.
In: Murray PR, Baron EJ, Jorgensen JH, Pfaller MA, Yolken RH. Manual of Clinical Microbiology, $8^{\text {th }}$ ed., American Society for Microbiology, Washington D.C., USA.

36. Shafazand S, Weinacker AB. Blood cultures in the Critical Care Unit: Improving Utilization and Yield. Chest 2002; 122: 1727-36.

37. Thomson RB, Miller JM. Specimen collection, transport, and Processing: Bacteriology. In: Murray PR, Baron EJ, Jorgensen JH, Pfaller MA e Yolken RH. Manual of Clinical Microbiology, $8^{\text {th }}$ ed., American Society for Microbiology, Washington D.C., USA.

38. Wasilauskas BL, Ellner PD. Presumptive identification of bacteria from blood cultures in four hours. $\mathrm{J}$ Infect Dis 1971; 124: 499-504.

39. Weinstein MP, Reller LB, Murphy JR, et al. The clinical significance of positive blood cultures: a comprehensive analysis of 500 episodes of bacteremia and fungemia in adults; I. Laboratory and epidemiological obeservations. Rev Infect Dis 1983; 5: 35-53.

40. Weinstein MP, Towns ML, Quartey SM, et al. The clinical significance of positive blood cultures in the 1990s: a prospective comprehensive evaluation of the microbiology, epidemiology, and outcome of bacteremia and fungemia in adults. Clin Infect Dis 1997; 24: 584-602.

\section{PROPOSTE PER L'ESECUZIONE DELL'EMOCOLTURA (SISTEMI IN BRODO)}

Il risultato ottimale di questo esame dipende da molteplici fattori fra cui principalmente il volume del campione, il momento del prelievo, il numero dei prelievi, l'accuratezza del prelievo (il metodo di disinfezione della cute), le caratteristiche del mezzo di coltura, la capacità del sistema analitico di evidenziare lo sviluppo batterico, l'interpretazione del risultato (patogeni vs contaminanti).

Di seguito vengono esaminate le principali variabili con indicazioni tratte dalla letteratura più recente ed autorevole.

\section{Fase pre-analitica}

\section{Tempo del prelievo}

Effettuare il prelievo prima del rialzo febbrile (6090 minuti prima) e, se questo non è prevedibile, allora prelevare all'inizio del rialzo febbrile. Effettuare il prelievo prima della prescrizione della terapia antimicrobica; in caso di terapia in corso, prima della somministrazione di antibiotico.

Protocolli di prelievo consigliati:

1) Sospetta sepsi meningite, osteomielite, artrite, polmonite, endocardite acuta, ecc. : due o tre prelievi diversi nell'arco di 30-60 minuti e prima di iniziare la terapia antimicrobica.

2) Sospetta endocardite sub-acuta: Come per l'acuta ma da ripetere eventualmente il giorno dopo.

3) Sospetta endocardite, sepsi ed altre cause di batteriemia in paziente sotto trattamento antibiotico. Due prelievi diversi nell'arco di 30-
60 minuti per tre giorni consecutivi e lontano dalla somministrazione del farmaco.

4) Febbre di origine sconosciuta (es. tifo, brucellosi): due prelievi diversi nell'arco di 30-60 minuti per tre giorni consecutivi

2. Numero di prelievi e intervallo tra prelievi Effettuare almeno due prelievi. Un solo prelievo può non evidenziare una batteriemia intermittente ed inoltre rende difficile interpretare il significato clinico dell'isolamento di certi microrganismi (possibili contaminanti cutanei).

Non esistono indicazioni sull" intervallo tra prelievi; molti Autori concordano sull'effettuare i prelievi a distanza di 10 '.

\section{Volume di sangue}

Esiste una relazione diretta fra volume di sangue prelevato e positività: nella maggior parte dei casi nell'adulto si usa prelevare una quantità di circa $10 \mathrm{ml}$ di sangue per flacone; in età pediatrica poiché la batteriemia presenta una carica microbica più elevata, si prelevano in genere da 1 a $5 \mathrm{ml}$ di sangue per flacone.

Deve esserci un rapporto ottimale fra volume del campione e volume del brodo di coltura (di solito 1:5) per cui non superare mai le quantità di sangue indicate sul flacone stesso.

\section{Quali brodi utilizzare?}

Nella normale routine, salvo accordi con il singolo reparto di degenza, fare inoculare, per ogni pre- 
lievo, un flacone per aerobi ed uno per anaerobi (il materiale è provvisto dai singoli laboratori a seconda della gamma di flaconi e ditte diagnostiche prescelte) e quando necessario ricorrere a flaconi pediatrici; non esistono evidenze che l'uso di flaconi specifici per miceti porti vantaggi di costo/beneficio.

\section{Modalità di prelievo:}

1) Selezionare un diverso punto per ogni prelievo.

2) Non aspirare il sangue da cateteri venosi o arteriosi a permanenza a meno che non sia possibile effettuare la puntura endovenosa o si sospetti una sepsi da catetere endovascolare (in questo caso prendere accordi con il laboratorio).

3) Disinfettare accuratamente la zona della puntura venosa con soluzione di disinfettante battericida iniziando in cerchi concentrici coprendo un' area di circa $4-5 \mathrm{~cm}$.

4) Lasciare asciugare e introdurre l'ago senza ripalpare la zona disinfettata; se fosse necessario palpare di nuovo la zona disinfettata, usare guanti sterili.

5) Disinfettare con uguale disinfettante il tappo di ogni bottiglia e lasciare asciugare.

6) Introdurre il sangue nella bottiglia e agitare bene per impedire la formazione del coagulo.

7) Porre sul flacone l'etichettatura prevista per identificare il paziente senza coprire eventuali codici a barre già stampati sul flacone stesso.

\section{Disinfettante cutaneo}

Sono spesso utilizzati: t,povidone iodio $10 \%$, tintura di iodio $2 \%$, clorexidina alcolica $0,5 \%$; gli ultimi due sono da preferirsi per l'azione più rapida (sufficiente un tempo di contatto di 15-30").

La disinfezione della cute è particolarmente importante per ridurre

l'inquinamento e contenere il numero dei falsi positivi che possono produrre aumenti del tempo di degenza, maggiori costi per la terapia, danno per il paziente e induzione di resistenze batteriche.

\section{Quesito diagnostico}

Il curante deve specificare nella richiesta (modulo cartaceo o via elettronica) l'esistenza o il sospetto di condizioni che possono richiedere modifiche nelle procedure di laboratorio (prolungamento del periodo di incubazione).

\section{Invio e conservazione}

Dopo il prelievo inviare subito le bottiglie in laboratorio o, se questo è impossibile, conservarle con le modalità proprie del sistema in uso in laboratorio.

\section{Fase analitica}

9. Accettazione dei campioni e loro incubazione Appena i flaconi pervengono in laboratorio, dopo il controllo di idoneità (biologicamente sicuri, adeguati in quantità, correttamente etichettati ecc.), se non ci sono i motivi previsti per respingerli (ricordare a questo proposito di avere regole scritte ben precise e precedentemente comunicate al clinico) vengono messi in incubazione con le modalità previste dalla ditta produttrice dei flaconi ed osservando le previste norme di contenimento del rischio infettivo. In caso di vistosa contaminazione esterna del contenitore e/o del foglio di accompagnamento, il campione sarà respinto perché potenzialmente pericoloso per il personale o adeguatamente disinfettato.

\section{Tempo di incubazione}

Per quanto riguarda i sistemi manuali l'incubazione consigliata, salvo le condizioni cliniche e microbiologiche che prevedono un prolungamento, è di sette giorni ricordando che, nonostante alcuni autori raccomandino colture cieche a 48 , 72 e a fine incubazione, le colture cieche durante ed a fine incubazione non sembrano giustificate alla luce delle analisi costi / benefici. Colture cieche non sono invece richieste quando si usino flaconi bifasici tipo "Castaneda".

Per quanto riguarda i sistemi assistiti da strumentazione la maggior parte degli autori suggerisce tempi di incubazione di 5 - 7 giorni, sempre escludendo i casi di necessario prolungamento per le ragioni viste sopra. La riduzione a 5 giorni può raramente comportare il mancato isolamento di possibili patogeni, ma riduce certamente anche la quota di contaminanti.

Non esistono precise indicazioni sui casi per cui si debba prolungare l'incubazione nè sulla durata della stessa. Per lo più si consigli di prolungare l'incubazione a due-quattro settimane nel sospetto di endocardite (gruppo HACEK ed altri batteri a lento sviluppo), di brucellosi e, secondo alcuni Autori, nel sospetto di micosi profonda o da anaerobi.

\section{Rilevazione dei segni di crescita}

Per i sistemi manuali deve essere prevista una ispezione dei flaconi per evidenziare la crescita almeno due volte durante il turno lavorativo; per i sistemi automatici il monitoraggio ovviamente è in continuo. Quando si usino sistemi automatici è opportuno stabilire momenti di esecuzione precisi nella giornata: ad esempio all'inizio e fine mattina e, nel pomeriggio.

La rilevazione della positività deve essere effet- 
tuata anche nei giorni festivi. Nel caso in cui, per carenza di risorse, questo non sia possibile si raccomanda di accordarsi con i reparti di degenza in modo che, in caso di gravi condizioni di pazienti con emocolture in corso, il clinico possa ricorrere al servizio di reperibilità del laboratorio.

\section{Procedure in caso di positività}

Ogni volta che vengono evidenziati segni di sviluppo (visivamente o segnalati dallo strumento), si deve procedere all'esame microscopico, alle subculture e, possibilmente anche all'esecuzione di test diretti di identificazione ed antibiogramma.

\section{a. Sottocolture}

Prendere i flaconi risultati positivi ed in cappa a flusso laminare preparare uno striscio prelevando una quantità di sangue/brodo per l'esecuzione della parte microscopica e colturale. Per evitare aghi e siringhe si suggerisce di utilizzare sistemi di raccordo per farfalle "vacutainer" che da una parte hanno un tubicino in plastica e dall'altra un ago. Inserire nel tappo di gomma la parte con l'ago e far uscire la goccia di brodo/sangue direttamente sul vetrino e piastre: in questo modo si evita di esporre al rischio di puntura accidentale il personale addetto a queste manipolazioni.

Sia dai flaconi per aerobi che per anaerobi devono essere inoculate:

- due piastre di agar sangue (una da incubare in aerobiosi ed una in anaerobiosi);

- una piastra di agar cioccolato arricchito (da incubare in $\mathrm{CO}^{2}$ )

Può essere utile anche l'aggiunta di piastre selettive per enterobatteri e agar CNA e Sabouraud (in particolare se lo si ritiene necessario per avere un orientamento che faciliti l'interpretazione delle colonie e/o se lo si ritiene necessario sulla base di quanto osservato al microscopio).

\section{b. Microscopia}

Da ogni flacone positivo deve essere effettuato un esame microscopico dopo colorazione di Gram per confermare la presenza di batteri o miceti e osservarne la morfologia ed i caratteri tintoriali. Alcune microbiologie effettuano lo screening con arancio di acridina, eseguendo poi il Gram sui positivi, altre colorano con arancio di acridina i vetrini che risultino negatiuvi al Gram.

Il referto dell'esame microscopico deve essere il più descrittivo possibile (esempio se la lettura è fatta da microbiologo esperto specificare streptococchi o stafilococchi piuttosto che cocchi gram positivi) e comunicato tem- pestivamente al medico curante. Attenzione però: deve essere chiarito al medico curante che microscopicamente non possiamo distinguere lo stafilococco aureo dagli altri stafilococchi per cui occorre molta prudenza nell'interpretazione del risultato (patogeno o contaminante?).

L'esame microscopico serve anche come guida all'esecuzione di eventuale identificazione ed antibiogramma "diretti".

\section{c. Identificazione diretta}

Non ci sono procedure approvate né standardizzate per questo test pertanto in questo caso l'uso di sistemi di qualunque genere viene lasciato alla responsabilità ed esperienza personale tenendo presente che una identificazione non ragionevolmente certa può fuorviare l'indirizzo terapeutico ed essere pertanto dannosa, per cui si consiglia la massima prudenza nell'interpretazione del risultato da comunicare al clinico.

\section{d. Antibiogramma diretto}

Neanche per questo test ci sono procedure standardizzate, ma in base all'esperienza personale ed a quella riportata dalla letteratura, si raccomanda fortemente di effettuarla perché può dare informazioni notevolmente utili in tempi relativamente brevi e comunque precedenti a quelli che saranno eseguiti con le metodiche standard dalle colonie isolate. Bisogna infatti ricordare che gli errori nei "diretti" eseguiti con il metodo della diffusione sono pochi e nella maggior parte dei casi sono "minor error".

\section{Fase post-analitica}

\section{Trasmissione dei risultati}

In caso di positività, è cura dei laureati del laboratorio avvisare tempestivamente (telefonicamente / fax / rete informatica) il medico di reparto dando le prime informazioni sul tipo di germe isolato in base alle caratteristiche tintoriali e morfologiche. Se usiamo il telefono (è auspicabile per avere uno scambio diretto di informazioni) dobbiamo registrare ora, data e il nome del medico a cui diamo l'informazione.

Successivamente (normalmente dopo 24 ore) in attesa del referto finale, vengono inviate al reparto (via tel., fax, ecc..) ulteriori informazioni sull'identificazione e antibiogramma dello stipite isolato.

Nel caso in cui nei giorni festivi il laboratorio sia chiuso si deve almeno specificare che se, per motivi di effettiva necessità dovuti alla gravità 
dello stato del paziente, sia necessario avere notizie di una emocoltura, si può telefonare al laureato reperibile per la Microbiologia.

\section{Interpretazione dei risultati}

\section{a. Risultati falsi negativi}

In caso di fondato sospetto di infezione accompagnata da batteriemia con emocolture negative (falsi negativi), prendere in considerazione le seguenti cause:

- Situazioni cliniche: Pregressa terapia antibiotica (60\% dei negativi), Uremia

- Microrganismi cosiddetti "difficili"
1) Batteri del gruppo HACEK (Haemophilus, Actinobacillus, Cardiobacterium, Eikenella, Kingella)

2) Abiotrophia: "Nutritionally variant streptococci"

3) Batteri a lento o lento sviluppo quali: Miceti, Brucella sp, Nocardia sp., Bartonella sp.

- Microrganismi coltivabili solo con metodiche specifiche e quindi da prelevare ed inviare al laboratorio con modalità diverse dalla normale routine, quali: Micobatteri, Leptospira sp. Legionella sp, Borrelia $s p$

- Microrganismi non coltivabili nei comuni terreni di coltura per batteri e pertanto evidenziabili mediante prove sierologiche, colture cellulari o, se disponibili, tecniche biomolecolari (Chlamydia sp, Coxiella burneti (febbre Q), altre Rickettsiae, Tropherina whippleri (Whipple Disease Bacillus)

\section{b. Risultati falsi positivi}

Positività del test legato a contaminazione del campione. Dal 30 al 50\% dei risultati positivi sono falsi positivi.

Come identificare i falsi positivi:

- aspetti clinici: decorso clinico non tipico, non riscontrata l'infezione primaria con lo stesso isolato,

- aspetti microbiologici: Unico isolamento da più flaconi di un microrganismo appartenente ad una delle specie che più facilmente danno inquinamento (vedi stafilococchi non aurei), positività per flora polimicrobica, positività che avviene dopo 5-6 giorni di incubazione,

Il giudizio di positività vera deriva quindi da molteplici fattori che devono tener conto dei giorni necessari alla positivizzazione del test, della presenza di positività in più flaconi, della classe di rischio clinico e della categoria di microrganismo.

\section{Stipiti isolati}

E' consigliabile, dopo la coltura, conservare i ceppi isolati almeno per un mese in attesa di altre eventuali richieste di esami da parte del clinico. Se possibile istituire una ceppoteca permanente con gli isolati in questa sede data la loro sicura importanza clinico-eziologica.

\section{Antonio Goglio}

A.O. Ospedali Riuniti di Bergamo

U.O. Microbiologia e Virologia

Largo Barozzi 1 - 24128 Bergamo

Tel. +039035 269573; Fax +039035266666

E-mail: agoglio@ospedaliriuniti.bergamo.it 